\title{
Cross-calibrating the energy scales of cosmic-ray experiments using a portable radio array
}

\author{
K. Mulrey, ${ }^{1, *}$ S. Buitink, ${ }^{1,2}$ A. Corstanje, ${ }^{1,2}$ K. D. de Vries, ${ }^{1}$ H. Falcke, ${ }^{2,3,4}$ B. M. Hare, ${ }^{5}$ \\ J. R. Hörandel, ${ }^{1,2,3}$ T. Huege,${ }^{1,6}$ G. K. Krampah, ${ }^{1}$ P. Mitra, ${ }^{1}$ A. Nelles, ${ }^{7,8}$ H. Pandya, ${ }^{1}$ \\ J. P. Rachen, ${ }^{1}$ E. Santiago, ${ }^{1}$ O. Scholten, ${ }^{9}$ R. Stanley, ${ }^{1}$ S. ter Veen,,${ }^{2,4}$ S. Thoudam, ${ }^{10}$ \\ T. N. G. Trinh ${ }^{11}$ and T. Winchen ${ }^{12}$ \\ ${ }^{1}$ Astrophysical Institute, Vrije Universiteit Brussel, Pleinlaan 2, 1050 Brussels, Belgium \\ ${ }^{2}$ Department of Astrophysics/IMAPP, Radboud University, P.O. Box 9010, 6500 GL Nijmegen, The \\ Netherlands \\ ${ }^{3}$ Nikhef, Science Park 105, 1098 XG Amsterdam, The Netherlands \\ ${ }^{4}$ Netherlands Institute of Radio Astronomy (ASTRON), Postbus 2, 7990 AA Dwingeloo, The Netherlands \\ ${ }^{5}$ University of Groningen, Kapteyn Astronomical Institute, Groningen, 9747 AD, Netherlands \\ ${ }^{6}$ Institut für Kernphysik, Karlsruhe Institute of Technology (KIT), P.O. Box 3640, 76021, Karlsruhe, \\ Germany \\ ${ }^{7}$ DESY, Platanenallee 6, 15738 Zeuthen, Germany \\ ${ }^{8}$ ECAP, Friedrich-Alexander-University Erlangen-Nürnberg, 91058 Erlangen, Germany \\ ${ }^{9}$ Interuniversity Institute for High-Energy, Vrije Universiteit Brussel, Pleinlaan 2, 1050 Brussels, Belgium \\ ${ }^{10}$ Department of Physics, Khalifa University, PO Box 127788, Abu Dhabi, United Arab Emirates \\ ${ }^{11}$ Department of Physics, School of Education, Can Tho University Campus II, 3/2 Street, Ninh Kieu \\ District, Can Tho City, Vietnam \\ ${ }^{12}$ Max-Planck-Institut für Radioastronomie, Auf dem Hügel 69, 53121 Bonn \\ E-mail: kmulrey@vub.be
}

\footnotetext{
*Presenter
} 
Different experiments use different techniques to detect and reconstruct cosmic-ray events, yielding different energy scales. Having a method to compare the energy scales of different experiments with minimal uncertainty is necessary in order to make meaningful comparisons of their spectra and composition measurements, which are used to create global models of cosmic-ray sources, acceleration and propagation. Comparing energy scales has proven to be difficult, given that uncertainties on energy measurements depend on the location, technique and equipment used. In this contribution we introduce a new radio-based technique which will be used to build a universal cosmic-ray energy scale. Radio detection provides a measure of the radiation energy in air showers, which scales quadratically with the electromagnetic energy. Once the local magnetic field strength is taken into account, radiation energy can be directly compared at different locations. A portable array of antennas will be built and deployed at various experiments, measuring radiation energy in conjunction with the host experiments' traditional air shower measurements. The energy measured at each location can then be directly compared via the contemporaneous radiation energy measurements. Using radiation energy to compare the energy scales eliminates uncertainties due to measurements being made at different locations, and using the same array at each site eliminates the uncertainties associated with the equipment and calibration. This will allow for a cross-calibration of the energy scales of different experiments with minimal uncertainty. Here we present the technique and report on the status of a prototype array that began taking data in January 2021.

$37^{\text {th }}$ International Cosmic Ray Conference (ICRC 2021)

July 12 th - 23rd, 2021

Online - Berlin, Germany 


\section{Introduction}

The cosmic-ray energy spectrum has a changing spectral index at the highest energies, which indicates a transition of sources from Galactic to extra-galactic, and eventually a suppression of cosmic-ray flux. In order to address open questions about the origin of the highest energy cosmic rays, it is critical to understand this region of the spectrum [1]. In this energy range different experiments have inconsistent energy scales, and it is necessary to shift the energy scales with respect to one another in order to align them to produce an overall spectral fit [2,3]. Comparing energy scales directly is difficult because experiments use different detection, calibration, and reconstruction techniques, all of which affect the overall scale and associated uncertainties.

Many methods are used to detect air showers indirectly. Particle detectors measure air shower particles that reach the Earth's surface, which can be used to estimate the total energy in the air shower. This method samples only a snapshot of the shower development, and furthermore, relies heavily on hadronic interaction models for the interpretation of data, which introduce large systematic uncertainties at high energies [4]. The fluorescence technique eliminates complications due to hadronic models, but the detectors are expensive to build and reconstruction requires good knowledge of atmospheric conditions.

Broadband radio emission is generated as air showers develop and has proven to be a very effective detection technique [5]. The radio emission is primarily due to the geomagnetically induced, time-varying transverse current that develops as the shower propagates $[6,7]$. The strength of this "geomagnetic" emission scales with the strength of the local geomagnetic field and the sine of the angle between the shower propagation and the geomagnetic field. A smaller "charge excess" contribution to the radio emission comes from the development of a charge excess in the shower front [8]. Radio emission is produced primarily by the electromagnetic components of the shower, and thereby bypasses uncertainties associated with hadronic interaction models [9]. Furthermore, the measured radio signals are integrated over the whole air shower, meaning measurements can be used to perform complete calorimetric energy reconstructions [10].

The total energy radiated by the air shower in the form of radio emission is called radiation energy. Once the radiation energy is adjusted for the strength of the local magnetic field and second order effects (such as the relative charge excess contribution), it becomes a universal quantity that can be directly compared between experiments [11]. When the radiation energy is found in conjunction with the air shower energy, as determined using an independent method, it can be used to compare the energy of cosmic rays detected at different locations. This allows for the direct comparison of energy scales between experiments [12]. An energy cross-calibration array will be build for this purpose and is presented in these proceedings.

\section{Concept}

Energy scales between experiments have already been successfully compared using radio techniques. KASCADE-Grande [13] and Tunka-133 [14] were compared using their radio extensions [15], although not using radiation energy, which was a limiting factor in the comparison. The fact that the same antennas were used at each location reduced the systematic uncertainties on the comparison. The energy scales of the particle detector installation at LOFAR [16] and Auger [17] 


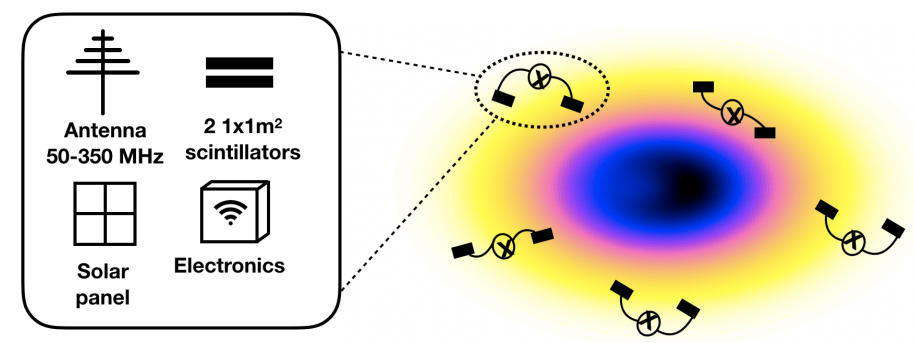

Figure 1: Energy cross-calibration array design. The array consists of 5 stations, each with an antenna, two scintillators, a solar panel, readout electronics and data storage.

were compared using radiation energy, but the antennas used at each site differed, adding substantial uncertainty to the comparison [12]. If a comparison could be made using the same detection system and using radiation energy, the systematic uncertainties on the comparison become minimal, and a meaningful, quantitative statement could be made about the energy scales of each experiment relative to one another. This concept is the basis for a project that aims to build a universal energy scale.

\section{Method}

This project has two main requirements: it must be able to measure radiation energy to high precision, and it must use the same detection system in multiple locations to minimize the systematic uncertainty on the comparison. A portable array of antennas, which can physically be moved to multiple locations, satisfies these criteria. The radiation energy can be determined using radio measurements, and the fact that the same antennas are used eliminates the majority of systematic uncertainties on the comparison. In this section we will present the array design and radiation energy reconstruction techniques.

\subsection{Array design}

The physics requirement of this array is that it can make radio measurements that lead to an accurate reconstruction of the radiation energy of a given event. In order to be used in a practical sense, there are other things to consider. First, the array has to be fully portable. This means we limit the number of array stations to five. This strikes a reasonable balance between portability and reconstruction ability, which will be discussed below. As the array is intended to be used at multiple sites, it would be impractical to base the design on any particular experiment or infrastructure, and so the array stations will also be fully autonomous. The stations are designed to be powered using solar panels, and data will be stored locally. Each station will operate independently and events will be correlated offline using timestamps. Triggering on radio events alone is always a challenge, especially when considering a design that should be applicable at any site. For this reason, each station will also include scintillator panels for triggering. Two panels at each station will look for coincident events and trigger radio readout. The spacing of the scintillator panels dictates the trigger rate, and can be adjusted accordingly. At a spacing of $20 \mathrm{~m}$, the trigger rate for a single station is on the order of $0.1 \mathrm{~Hz}$ which mitigates concerns about dead time and makes long term 
storage manageable. The array design is shown in Fig. 1. Since the stations are all independent, their relative spacing can be adjusted to optimize detection in the energy range of interest.

The antennas used will be of the SKALA design and measure between 50 and $350 \mathrm{MHz}$ [18]. These are well characterized and used by a number of experiments $[19,20]$. The scintillators have been recycled from the KASCADE experiment [13], and the digitizing electronics for the radio readout have been provided by the CODALEMA experiment [21].

\subsection{Radiation energy reconstruction}

Having an good reconstruction of the radiation energy of an event is critical to this project. We will build on the reconstruction techniques demonstrated by AERA [22] and proposed by ARIANNA [23]. The AERA group demonstrated that it was possible to reconstruct radiation energy with just 3-5 antennas in the $30-80 \mathrm{MHz}$ range using a technique that compares the measured fluence to a two dimensional lateral distribution function that describes the radio footprint [10]. Using this method, a resolution of $22 \%$ is achieved for the whole data set, which increases to $17 \%$ for events with 5 illuminated antennas. For events with 3 or more illuminated antennas, we expect to be able to reconstruct radiation energy at least that well. One advantage the cross-calibration array has over the traditional $30-80 \mathrm{MHz}$ experiments is added bandwidth. Using the wider $50-350 \mathrm{MHz}$ bandwidth retains the higher signal regime at the lower frequencies, while also including the higher frequencies that sharpen the features of the radio footprint. This will allow us to better constrain the position of the antenna in the footprint. An example of the difference between a $30-80 \mathrm{MHz}$ footprint and a $50-350 \mathrm{MHz}$ footprint for the same event is shown in the right panel of Fig. 2. Additionally, in event reconstruction, the cross-calibration array has the opportunity to use some information from the host experiment. For every event detected by the cross-calibration array, the same event has (most likely) also been seen by the host experiment, which will have done its own reconstruction, including core position and direction. Using this information will allow for the reconstruction of events seen in just a few antennas without biasing the results.

The ARIANNA experiment has shown through a simulation study that it is possible to reconstruct radiation energy using just one broadband antenna [24]. Using a set of realistic Monte Carlo events and an $80-300 \mathrm{MHz}$ bandwidth, they have reconstructed radiation energy with a resolution better than $15 \%$. This is possible because the frequency content of the signal is different at different radii from the core in the radio footprint (left panel of Fig. 2), allowing for the determination of the antenna's position in the footprint. With more than one antenna in the illuminated area, and with potential knowledge of the shower core, we expect to able to achieve at least $15 \%$ resolution in radiation energy.

\section{Implementation}

A prototype energy cross-calibration array has been built at the Vrije Universiteit Brussel. This installation has been valuable for developing the prototype and characterizing backgrounds. An image of the prototype is shown on the right side of Fig. 3. The power spectra of a detected cosmic-ray event is shown on the left. Later in 2021 we plan to deploy the full cross-calibration array at the LOFAR site and take data for approximately 6 months. During this time, it is expected that on the order of a few hundred reconstructable events will be detected both by the portable 

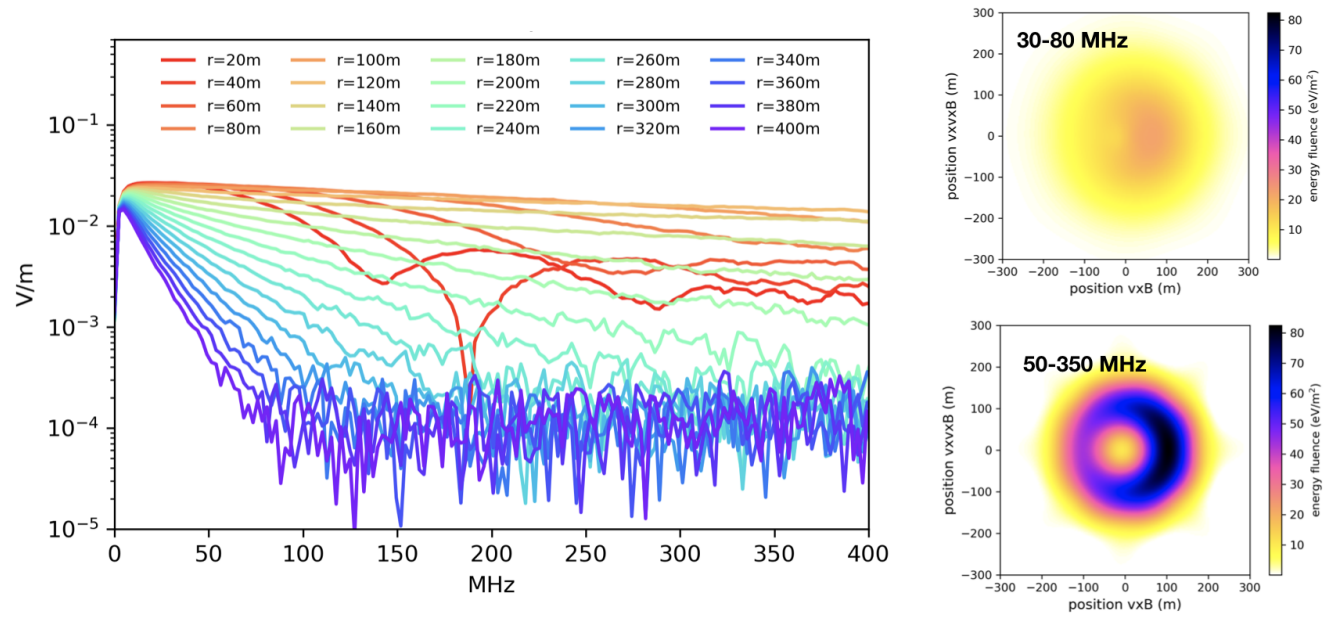

Figure 2: Left: Frequency content for a cosmic-ray of energy $10^{17} \mathrm{eV}, X_{\max }=640 \mathrm{~g} / \mathrm{cm}^{2}$, and with a zenith angle of $33^{\circ}$ on the $\mathrm{vxvxB}$ axis. Each color represents an antenna position at different radii from the core. Right: Energy fluence for the same cosmic ray, for a frequency band of $30-80 \mathrm{MHz}$ (top) and $50-350 \mathrm{MHz}$ (bottom).

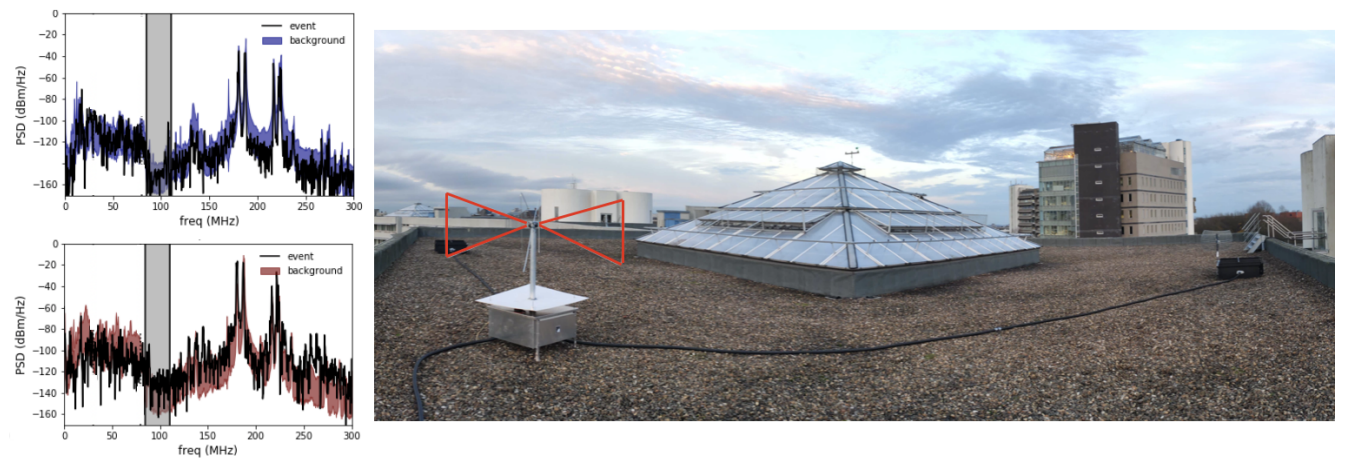

Figure 3: Left: Power spectra of a sample cosmic-ray event. The average background is show as the colored background. The region of a FM bandpass filter is shown in gray. Right: Prototype array station located on the roof of the VUB. The antenna is highlighted in the center, with scintillators housed in black boxes on either side. The roof prototype uses CODALEMA butterfly antennas, while the field setup will use SKALA antennas.

array and by LOFAR. Then, the array will be moved to the Auger site, presumably in the densely instrumented area, where again data will be collected for 6 months to a year. The radiation energy for each event will be determined, and a relation drawn between the radiation energy and reconstructed cosmic-ray energy for each experiment (in the same was as was done in [12]). With this information, the difference between the LOFAR and Auger energy scales will be quantitatively determined with minimal uncertainties. After demonstrating the effectiveness of this technique, the cross-calibration array will be moved to other experiment sites.

There are also plans to use the design of this cross-calibration array in the prototype of the Radar Echo Telescope for Cosmic Rays (RET-CR) project [25]. The antenna stations will comprise a complementary surface component to an in-ice radar detection system. The surface array will 
both provide a trigger for radar readout and also an independent event reconstruction method. More information can be found in $[25,26]$.

\section{Summary}

The energy cross-calibration array will allow for the direct comparison of the energy scales of different experiments using the universal measurement of radiation energy. The comparison will have very small systematic uncertainties, as the same detection system will be used in each place. This quantitative understanding of the different energy scales between experiments is critical to address open questions about the origin of the highest energy cosmic rays. A prototype array has been built and is ready for deployment later this year.

\section{References}

[1] J. Blümer, R. Engel, and J. R. Hörandel. Cosmic Rays from the Knee to the Highest Energies. Prog. Part. Nucl. Phys., 63:293-338, 2009.

[2] J. Hörandel. On the knee in the energy spectrum of cosmic rays. Astropart. Phys., 19:193-220, 2003.

[3] H. P. Dembinski, R. Engel, A. Fedynitch, T. Gaisser, F. Riehn, and T. Stanev. Data-driven model of the cosmic-ray flux and mass composition from $10 \mathrm{GeV}$ to $10^{11} \mathrm{GeV}$. PoS, ICRC2017:533, 2018.

[4] R. Engel, D. Heck, and T. Pierog. Extensive air showers and hadronic interactions at high energy. Ann. Rev. Nucl. Part. Sci., 61:467-489, 2011.

[5] H. Falcke et al. Detection and imaging of atmospheric radio flashes from cosmic ray air showers. Nature, 435:313-316, 2005.

[6] F. D. Kahn and I. Lerche. Radiation from cosmic ray air showers. R. Soc. Lond. A, 289, 1966.

[7] K. Werner and O. Scholten. Macroscopic Treatment of Radio Emission from Cosmic Ray Air Showers based on Shower Simulations. Astropart. Phys., 29:393-411, 2008.

[8] G. Askaryan. Excess negative charge of an electron-photon shower and its coherent radio emission. JETP, 14:441-443, 1962.

[9] T. Huege. Radio detection of cosmic ray air showers in the digital era. Physics Reports, 620:1-52, 2016.

[10] A. Aab et al. Measurement of the Radiation Energy in the Radio Signal of Extensive Air Showers as a Universal Estimator of Cosmic-Ray Energy. Phys. Rev. Lett., 116(24):241101, 2016.

[11] C. Glaser, M. Erdmann, J. R. Hörandel, T. Huege, and J. Schulz. Simulation of Radiation Energy Release in Air Showers. JCAP, 1609(09):024, 2016. 
[12] K. Mulrey et al. On the cosmic-ray energy scale of the LOFAR radio telescope. JCAP, 11:017, 2020.

[13] T. Antoni et al. The cosmic-ray experiment KASCADE. Nuclear Instruments and Methods A, 513:490, 2003.

[14] S.F. Berezhnev et al. The Tunka-133 EAS Cherenkov light array: status of 2011. Nucl. Instrum. Meth. A, 692:98-105, 2012.

[15] W.D. Apel et al. A comparison of the cosmic-ray energy scales of Tunka-133 and KASCADEGrande via their radio extensions Tunka-Rex and LOPES. Phys. Lett. B, 763:179-185, 2016.

[16] S. Thoudam et al. LORA: A scintillator array for LOFAR to measure extensive air showers. Nucl.Instrum.Meth, A767:339-346, 2014.

[17] Alexander Aab et al. The Pierre Auger Cosmic Ray Observatory. Nucl. Instrum. Meth. A, 798:172-213, 2015.

[18] E. de Lera Acedo, N. Razavi-Ghods, N. Troop, N. Drought, and A.J. Faulkner. SKALA, a log-periodic array antenna for the SKA-low instrument: design, simulations, tests and system considerations. Experimental Astronomy, 39:567-594, 2015.

[19] A. Balagopal V., A. Haungs, T. Huege, and F. G. Schroeder. Search for PeVatrons at the Galactic Center using a radio air-shower array at the South Pole. Eur. Phys. J. C, 78(2):111, 2018. [Erratum: Eur.Phys.J.C 78, 1017 (2018), Erratum: Eur.Phys.J.C 81, 483 (2021)].

[20] M.G. Labate, P. Dewdney, R. Braun, M. Waterson, and J. Wagg. The SKA low-frequency telescope: performance parameters and constraints on the array configuration. In 11th European Conference on Antennas and Propagation (EUCAP), pages 2259-2263, 2017.

[21] D. Ardouin et al. Radio-Detection Signature of High Energy Cosmic Rays by the CODALEMA Experiment. Nucl. Instrum. Meth., A555:148, 2005.

[22] J. Schulz. Status and Prospects of the Auger Engineering Radio Array. PoS, ICRC2015:615, 2016.

[23] Stuart A. Kleinfelder. Design of the Second-Generation ARIANNA Ultra-High-Energy Neutrino Detector Systems. In 2015 IEEE Nuclear Science Symposium and Medical Imaging Conference, 112015.

[24] Christoph Welling, Christian Glaser, and Anna Nelles. Reconstructing the cosmic-ray energy from the radio signal measured in one single station. JCAP, 10:075, 2019.

[25] S. Prohira, K.D. de Vries, et al. The Radar Echo Telescope for Cosmic Rays (RET-CR): Pathfinder Experiment for a Next-Generation Neutrino Observatory. PoS, ICRC2021, 2021.

[26] R. Stanley et al. Simulation and Optimisation for the Radar Echo Telescope for Cosmic Rays. PoS, ICRC2021, 2021. 